Document downloaded from:

http://hdl.handle.net/10251/61428

This paper must be cited as:

Carrillo, Al.; Schmidt, LC.; Marín García, ML.; Scaiano, JC. (2014). Mild synthesis of mesoporous silica supported ruthenium nanoparticles as heterogeneous catalysts in oxidative Wittig coupling reactions. Catalysis Science and Technology. 4(2):435-440. doi:10.1039/c3cy00773a.

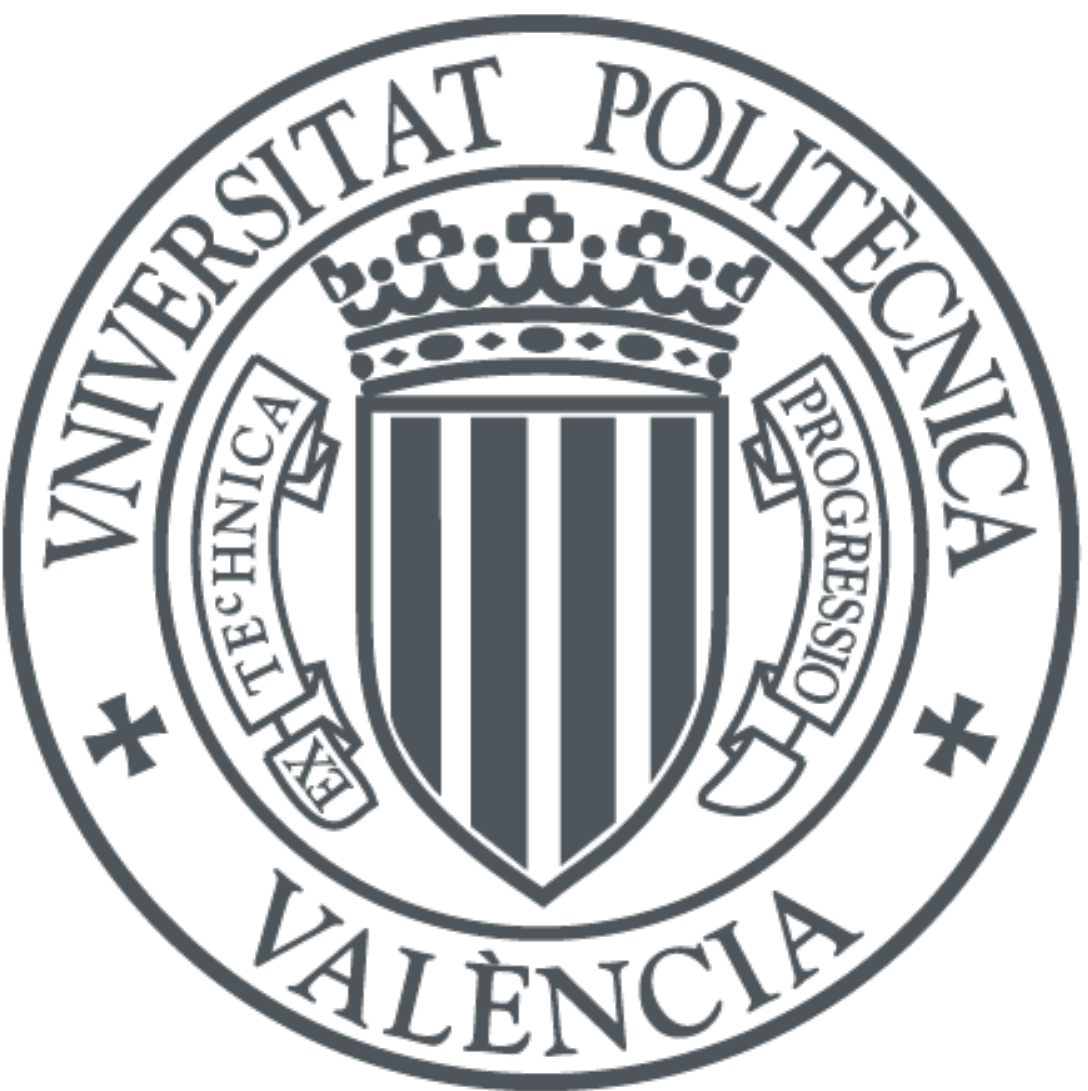

The final publication is available at

http://dx.doi.org/10.1039/c3cy00773a

Copyright Royal Society of Chemistry

Additional Information 


\title{
Mild synthesis of Mesoporous silica supported ruthenium nanoparticles as heterogeneous catalysts in oxidative Wittig coupling reactions
}

\author{
Adela I. Carrillo, ${ }^{a}$ Luciana C. Schmidt, ${ }^{a}$ M. Luisa Marín, ${ }^{\text {a,b }}$ and Juan C. Scaiano ${ }^{\text {a* }}$ \\ A new efficient approach for the in-situ synthesis of anchored ruthenium nanoparticles (RuNP) in three \\ different kinds of mesoporous silica materials MCM-41, SBA-15 and HMS has been developed. Solids \\ have been synthesized under very mild conditions from $\mathrm{RuCl}_{3} \cdot \mathrm{H}_{2} \mathrm{O}$ salt reduced in one hour at room \\ temperature in the mesoporous silicas previously grafted with aminopropyltriethoxisilane (APTES). \\ ${ }_{10}$ Well-dispersed ruthenium nanoparticles, with average size of $3 \mathrm{~nm}$, anchored onto the silica network by \\ the APTES were obtained. These materials, with molar ratios $\mathrm{Si} / \mathrm{Ru}=40$, were found to be catalytically \\ active and selective in the alcohol oxidation-Wittig olefination. Interestingly, while the reaction occurs \\ from the alcohol, control experiments suggest that the aldehyde (the common Wittig substrate) is not \\ involved.
}

\section{${ }_{15}$ Introduction}

The development of sustainable routes for the large scale production of fine chemicals, i.e., cost-effective and environmentally friendly, is one of the major current concerns at the industrial level. ${ }^{1}$ The use of supported metal nanoparticles 20 allows the combination of increased efficiency from nanoparticle catalysts, with the advantages of heterogeneous supports, leading to a 'green catalytic process', with higher selectivity, conversion and easy catalyst recovery. ${ }^{2}$

The design of the catalyst is a key step in the development of a 25 sustainable catalytic reaction. Among the most crucial issues in the preparation of a nanocatalyst is avoiding agglomeration of the nanoparticles as well as leaching from the active sites of the support; this can be achieved by anchoring the nanoparticles to the support surface.

30 Mesopororus silicas such MCM-41 are attractive catalyst supports because they present high surface areas where the active sites can be highly dispersed. Different strategies have been successfully employed to introduce catalytic active sites into mesoporous silicas including ion exchange, chemical vapor 35 deposition or impregnation. ${ }^{3,4}$ However, the interaction between the active site and the support is frequently very weak. This may cause leaching of the active sites into the reaction media, potentially leading to a decrease in the catalytic activity of the material. To overcome this issue, more recently co-condensation

40 or chemical grafting methods to covalently bond the active species into mesoporous materials are being reported. For example, co-condensation method has been used to incorporate palladium nanoparticles into a silica matrix by functionalizing the nanoparticles with alkoxysilanes and then co-polymerizing them

45 with tetraethoxysilanes in the presence of cationic surfactants via basic-catalyzed hydrolysis. ${ }^{5}$ Recently, the synthesis of an hybrid mesoporous material with a molybdenum complex covalently attached was reported ${ }^{6}$ by using either post synthesis grafting or co-condensation approaches.

50 Supported ruthenium catalysts, mostly obtained by impregnation have emerged as a new family of versatile catalysts for different chemical reactions. In fact, they have been employed in industrial processes for the synthesis of paraffins ${ }^{7}$, methanation of $\mathrm{CO}^{8}$ or in the hydrogenation of benzene to cyclohexane ${ }^{9}$.

${ }_{55}$ Supported ruthenium nanoparticles (SRuNP) have been found to have very good catalytic activity towards synthesis of ammonia ${ }^{10}$ or hydrogenation of monoaromatics. ${ }^{11}$

In the present work we take advantage of the activity of RuNP and mesoporous silica to prepare three new hybrid materials and ${ }_{60}$ explore their catalytic properties. The one-pot alcohol oxidationWittig reaction producing $\alpha, \beta$-unsaturated esters was chosen to test the catalytic activity of the materials as it is the most commonly used method for the synthesis of alkenes. ${ }^{12}$ The new materials showed good catalytic activity in the Wittig olefination 65 of benzyl alcohols. ${ }^{13}$ The most interesting point was that reaction occurs without the intermediacy of free aldehyde and with a high selectivity to the $E$ product. These new solids have been prepared by grafting as follows: first, APTES was anchored onto the surface of MCM-41, SBA-15 or HMS type silicas. Then an 70 aqueous solution of the $\mathrm{RuCl}_{3}$ was stirred in the presence of the solids at room temperature for $1 \mathrm{~h}$. The covalently bonded RuNP were fully characterized by BET surface area, pore size distribution, X-ray diffraction (XRD), transmission electron microscopy (TEM), X-ray photoelectron spectroscopy (XPS) and 75 inductively coupled plasma mass spectrometry (ICP).

\section{Experimental}

Materials 
Tetraethylorthosilicate (TEOS, 98\%) was used as silica precursor. Cetyltrimethylammonium bromide $\left(\mathrm{C}_{16} \mathrm{TAB}, 96 \%\right)$, pluronic (P123) and dodecylamine (DA, 98\%) were used as structuredirecting agents. Aqueous ammonia solution $\left(\mathrm{NH}_{4} \mathrm{OH}, 30 \%\right)$, 5 hydrochloric acid and APTES were also used in the synthetic protocol to obtain the final mesoporous materials. $\mathrm{RuCl}_{3} \cdot \mathrm{xH}_{2} \mathrm{O}$ was used as ruthenium source; methyl(triphenylphosphoranylidene) acetate, benzyl alcohol, 1,1'binaphthyl and the other primary alcohols were purchased

10 from Aldrich and used as received. Anhydrous toluene (spectroscopy grade) was used for the reactions.

\section{Preparation of mesoporous supports}

\section{Synthesis of MCM-41}

Synthesis of MCM-41 type silica was performed following 15 published method. ${ }^{6}$ Briefly, in a typical procedure, $0.44 \mathrm{~g}$ of $\mathrm{C}_{16} \mathrm{TAB}$ were dissolved in a $35 \mathrm{mM} \mathrm{NH}_{4} \mathrm{OH}$ solution $(41.92 \mathrm{~mL})$ at $40^{\circ} \mathrm{C}$. Then, $2.33 \mathrm{~mL}$ TEOS were added to the solution to achieve a molar composition of the synthesis gel of $1 \mathrm{SiO}_{2}: 0.12$ $\mathrm{C}_{16} \mathrm{TAB}: 1.41 \mathrm{NH}_{4} \mathrm{OH}: 280 \mathrm{H}_{2} \mathrm{O}$. The gel was then transferred to 20 a $100 \mathrm{~mL}$ Teflon-lined stainless steel autoclave and heated at $80^{\circ} \mathrm{C}$ under hydrothermal conditions for $24 \mathrm{~h}$. After cooling at room temperature, the solid obtained was washed with water and ethanol, filtered off, and air-dried overnight. Finally, the surfactant was removed by calcination at $550^{\circ} \mathrm{C}$ for $8 \mathrm{~h}\left(2^{\circ} \mathrm{C} \mathrm{min}-\right.$ ${ }_{25}{ }^{1}$ ) under static air atmosphere.

\section{Synthesis of SBA-15}

SBA-15 type silica was prepared according to a procedure described elsewhere. ${ }^{14}$ In a typical synthesis, $2 \mathrm{~g}$ of pluronic (P123) were dispersed in $15 \mathrm{~mL}$ of water and $60 \mathrm{~mL}$ of $2 \mathrm{M} \mathrm{HCl}$ 30 solution. Then, $4.66 \mathrm{~mL}$ of TEOS were added to the solution with stirring. This gel mixture was continuously stirred at $40^{\circ} \mathrm{C}$ for 24 $\mathrm{h}$ and finally precipitated in a Teflon-lined autoclave at $100^{\circ} \mathrm{C}$ for $48 \mathrm{~h}$. Then, the solid was filtered, washed with deionized water, dried in air at room temperature and finally calcined at $550^{\circ} \mathrm{C}$ 35 under static air conditions for $8 \mathrm{~h}\left(2^{\circ} \mathrm{C} \mathrm{min}^{-1}\right)$ in order to remove the surfactant.

Synthesis of hexagonal mesoporous silica (HMS)

Hexagonal mesoporous silica was synthesized according to the procedure reported by Zhang et al. ${ }^{15}$ Thus, $2 \mathrm{~mL}$ of TEOS were 40 added dropwise to a stirred mixture containing $10.5 \mathrm{~mL}$ of $\mathrm{H}_{2} \mathrm{O}, 9$ $\mathrm{mL}$ of absolute ethanol, and $1 \mathrm{~g}$ of dodecylamine. The resulting mixture was stirred at room temperature for $18 \mathrm{~h}$. The obtained white solid was filtered, washed several times with water and ethanol, filtered off, and air-dried overnight. Finally, the ${ }_{45}$ surfactant was removed by calcination at $550^{\circ} \mathrm{C}$ for $8 \mathrm{~h}\left(2^{\circ} \mathrm{C} \mathrm{min}\right.$ ${ }^{1}$ ) under static air atmosphere.

\section{Preparation of SRuNP}

First, $1.5 \mathrm{~g}$ of mesoporous silica solid, MCM-41, SBA-15 or HMS, were dehydrated in an oven at $473 \mathrm{~K}$ for $2 \mathrm{~h}$. Then, $50 \mathrm{~mL}$ 50 of anhydrous toluene were added to the activated materials and this mixture was stirred for $1 \mathrm{~h}$ in order to obtain a homogeneous dispersion followed by the addition of $0.5 \mathrm{~mL}$ APTES and refluxed overnight. Finally, the white solid (silica-APTES) obtained was filtered, washed with fresh toluene and acetone and 55 air-dried.

Second, ruthenium chloride $\left(\mathrm{RuCl}_{3} \cdot \mathrm{xH}_{2} \mathrm{O}, 9 \mathrm{mg}\right)$ was added to an aqueous mixture $(110 \mathrm{~mL})$ of the corresponding silica-APTES material $(1.5 \mathrm{~g})$. After stirring for $1 \mathrm{~h}$ at room temperature, the grey solids obtained were filtered and washed several times with 60 water to remove the unreacted salt. The air-dried new SRuNP (2.3-2.5 wt \% Ru, ICP determined) were denoted as Ru@MCM, Ru@SBA and Ru@HMS.

\section{Catalysis tests}

The catalysis tests were performed using the new mesoporous 65 silica materials. Typically, a mixture of benzyl alcohol $(0.1 \mathrm{~mL}$, $1.0 \mathrm{mmol}$ ), methyl(triphenylphosphoranylidene) acetate (334 mg, $1.0 \mathrm{mmol})$ and $50 \mathrm{mg}$ of SRuNP $(1.2 \mathrm{~mol} \% \mathrm{Ru})$ in $2 \mathrm{~mL}$ of toluene was stirred for $24 \mathrm{~h}$ at $80^{\circ} \mathrm{C}$ under an oxygen atmosphere. The yields were determined by GC-MS after filtration and 70 addition of 1,1'-binaphthyl as internal standard.

\section{Instrumentation}

\section{Nitrogen adsorption-desorption isotherms}

The textural properties of the solids were determined from $\mathrm{N}_{2}$ adsorption at $77 \mathrm{~K}$ in an AUTOSORB-6 apparatus. The samples 75 were previously degassed for $4 \mathrm{~h}$ at $373 \mathrm{~K}$ at $5 \times 10^{-5}$ bar. The adsorption branch was used to determine the pore size distribution using the Barret-Joyner-Helender (BJH) method. The surface area was determined using the multipoint BET method in the $0.05-0.30$ relative pressure ranges. Mesopore volume was 80 measured at the plateau of the adsorption branch of the nitrogen isotherm, $\mathrm{P} / \mathrm{Po}=0.8 .{ }^{6}$ Gas adsorption at higher $\mathrm{P} / \mathrm{Po}$ is mainly due to interparticle condensation.

\section{$\mathrm{X}$-ray diffraction studies}

Small-angle powder X-ray diffraction (XRD) analysis was 85 carried out with a Rigaku Ultima IV diffractometer using a CuKa radiation $(\mathrm{k}=1.541836 \AA)$, operating at $40 \mathrm{kV}$ and $30 \mathrm{~mA}$, at a scanning velocity of $0.03^{\circ} \mathrm{min}^{-1}$ in the $0.7^{\circ}<2 \theta<10^{\circ}$ range.

\section{TEM analysis}

The morphology of the mesoporous materials was characterized 90 by transmission electron microscopy (TEM). TEM analyses were carried out on a JEM-2010 microscope (JEOL, 200 kV, $0.14 \mathrm{~nm}$ resolution). For this purpose, samples were prepared from a sonicated suspension of the material in ethanol on a carboncoated copper grid. The digital analysis of the TEM micrographs 95 was done using DigitalMicrographTM 3.6.1 by Gatan.

\section{X-ray photoelectron spectroscopy (XPS)}

The electronic state of ruthenium species was determined by XPS using a SPECS spectrometer with a MCD-9 detector using a monochromatic $\mathrm{Al}(\mathrm{K} \alpha=1486.6 \mathrm{eV}) \mathrm{X}$-ray source. Spectra were 100 recorded using an analyzer pass energy of $50 \mathrm{~V}$, an $\mathrm{X}$-ray power of $200 \mathrm{~W}$, and under an operating pressure of 10 mbar. Spectra treatment was performed using the CASA software. Binding energies (BE) were referenced to the $\mathrm{C} 1 \mathrm{~s}$ peak at $283.3 \mathrm{eV}^{16}$

Inductively coupled plasma optical emission spectroscopy 105 (ICP-AES)

Metal loading was determined by ICP-AES on a Perkin-Elmer Analyst 300 absorption apparatus and plasma ICP Perkin Elmer $40.25 \mathrm{mg}$ of every sample were digested in $1 \mathrm{~mL} \mathrm{HF}$ during $12 \mathrm{~h}$ prior to analysis by ICP-AES.

\section{Reusability}

To check the reusability of the hybrid materials, they were washed with toluene, filtered out and heated in the oven up to 90 ${ }^{\circ} \mathrm{C}$ overnight. GC analysis of the last aliquot corroborated the absence of reaction products. 


\section{Results}

\section{Catalysts characterization}

Figure 1 compares the X-ray diffraction patterns of MCM-41,

SBA-15 and HMS before and after their functionalization with

5 anchored RuNP. In the patterns of MCM-41 type materials

(Figure 1top), a dominant (100) peak with small (110) and (200)

reflections is normally attributed to the 2D-hexagonal structure $(p 6 m m) .{ }^{17}$ Aminosilane grafting to $\mathrm{MCM}-41$ and further incorporation of RuNP caused a considerable decrease in the 10 XRD intensity.

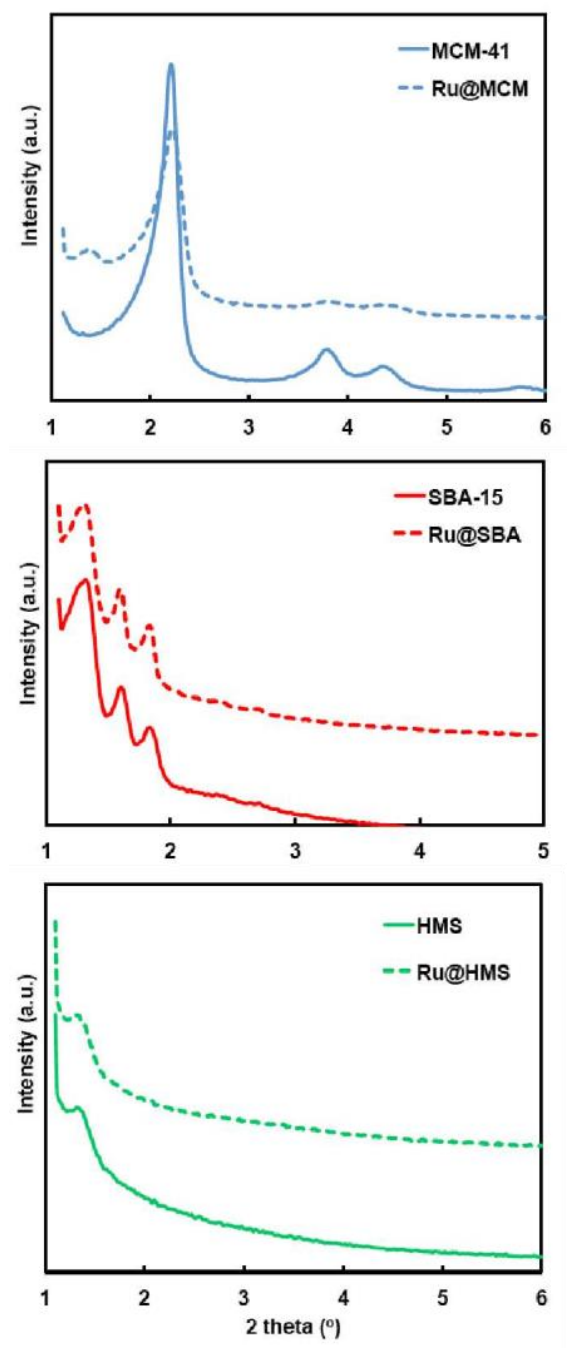

Figure 1. Low angle XRD patterns of pure silicas MCM-41 (top) SBA-15 (middle) and HMS (bottom) and their corresponding SRuNP materials.

15 TEM images of the SRuNP are shown in Figure 2. Ru@MCM and Ru@SBA reveal the hexagonal mesoporous arrangement typical for these materials, even after incorporation of RuNP, while in the case of Ru@HMS a disordered and wormhole mesostructure was shown ${ }^{15}$. Figure 2 also reveals that RuNP are 20 confined and highly dispersed into the channels of the mesoporous silica and confirm that these materials maintain the 2D-hexagonal mesopore arrangement of the pure MCM-41. The SBA-15 family of materials showed three well-defined peaks at $2 \theta$ values between 1 and $8^{\circ}$ (Figure 1 midle) that can be indexed
25 as (100), (110) and (200) Bragg reflections, typical of hexagonal (p6mm) SBA-15. ${ }^{18}$ In these materials, both the intensity and resolution of the peaks are not decreased by anchoring RuNP, probably because the size of the RuNP ( $c a .3 \mathrm{~nm}$ ) does not affect the pores of SBA-15 (ca.9 nm). HMS and Ru@HMS patterns

30 show a single low-angle diffraction peak characteristic of a wormhole framework ${ }^{19}$.

The new hybrid materials exhibited type IV isotherms with a distinctive nitrogen uptake due to the capillary condensation of nitrogen inside of mesopores (Figure 3). ${ }^{3}$ Further, each material 35 showed its characteristic features; for instance, MCM-41 solids showed a sharp increase in the adsorbed volume at $\mathrm{P} / \mathrm{Po}=0.2-0.4$ because of the 2-3 nm diameter pores. ${ }^{20}$ Otherwise, SBA-15 type materials showed an abrupt step at higher relative pressures (P/Po $=0.6-0.8)$ as expected for bigger pore size silicas. ${ }^{18}$ Finally, HMS

40 solids did not show an abrupt step in their isotherms profiles due to the broad pore size distributions ${ }^{3}$ Table 1 summarizes $\mathrm{BJH}$ pore size distributions calculated from the adsorption branches of the isotherms and BET surface areas of the new solids. As expected, in all cases both the pore diameter and the surface area

45 decreased after the incorporation of the anchored RuNP into the mesopores.
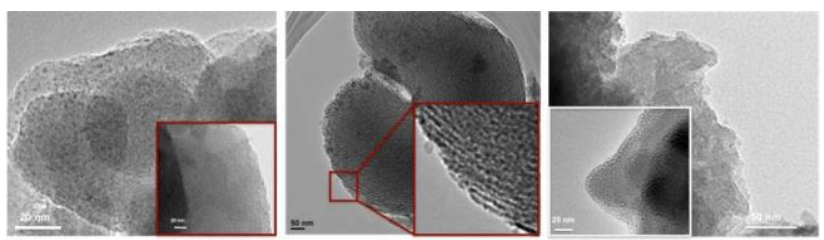

Figure 2. TEM images of the hybrid materials Ru@MCM (a),

50 Ru@SBA(b) and Ru@HMS (c).

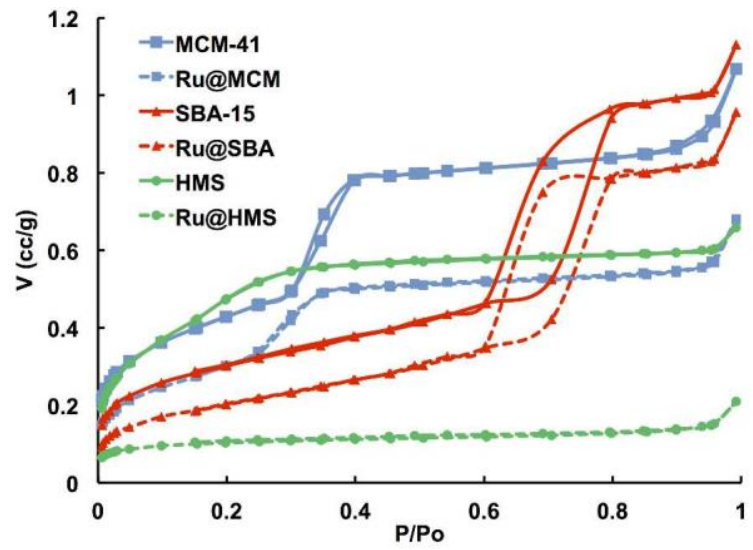

Figure 3. Nitrogen adsorption-desorption isotherms of the synthesized materials: MCM-41, Ru@MCM, SBA-15, Ru@SBA, HMS, and Ru@HMS.

55 Additional efforts were made to characterize the oxidation state of the SRuNP. Since the XRD experiments were inconclusive, XPS were performed on the all new materials, including the MCM-APTES that was used as standard. According to the XPS spectra (Figure 4) ( $\mathrm{Ru} 3 \mathrm{~d}_{5 / 2}$ at $280.4 \mathrm{eV}$, and $\mathrm{Ru} 3 \mathrm{p}_{3 / 2}$ 60 at $462.0 \mathrm{eV}$, respectively) demonstrated that the ruthenium particles were at least partially in a zero oxidation state in accordance with the literature, although other states are also likely to be found. ${ }^{16}$ 
Table 1. Textural properties and ruthenium loading of the new catalysts.

\begin{tabular}{|c|c|c|c|c|}
\hline Sample & $\begin{array}{c}d_{\mathrm{p}}^{B J H, \mathrm{a}} \\
(\mathrm{nm})\end{array}$ & $\begin{array}{c}\mathrm{A}_{B E T}^{\mathrm{b}} \\
\left(\mathrm{m}^{2} / \mathrm{g}\right)\end{array}$ & $\begin{array}{l}\mathrm{Vp}^{B J H, \mathrm{c}} \\
(\mathrm{cc} / \mathrm{g})\end{array}$ & $\begin{array}{c}\text { Metal } \\
\text { loading } \\
\text { wt } \%^{\mathrm{d}}\end{array}$ \\
\hline MCM-41 & 3.0 & 995 & 1.1 & ---- \\
\hline Ru@MCM & 2.4 & 740 & 0.7 & 2.3 \\
\hline SBA-15 & 9.0 & 785 & 1.2 & ---- \\
\hline Ru@SBA & 8.7 & 430 & 0.8 & 2.5 \\
\hline HMS & 2.0 & 700 & 0.7 & ----- \\
\hline Ru@HMS & $2.0-4.0$ & 190 & 0.4 & 2.3 \\
\hline
\end{tabular}

${ }^{a}$ Average mesopore diameters were estimated from the adsorption branch of the nitrogen isotherm using the BJH method. ${ }^{b}$ The BET surface area was estimated by multipoint BET method using the adsorption data in the 5 relative pressure $\left(\mathrm{P} / \mathrm{P}_{0}\right)$ range of $0.05-0.30 .{ }^{c}$ Mesopore volume from the isotherms at the relative pressure of $0.8 .{ }^{d}$ Ruthenium amount determined by ICP analysis.

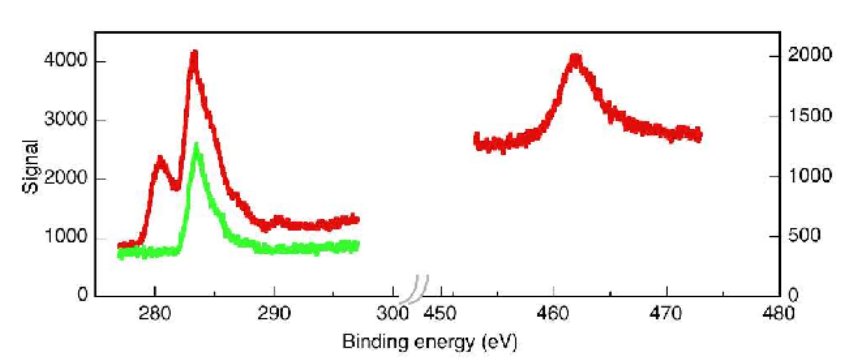

Figure 4: XPS spectra showing the $\mathrm{C} 1 \mathrm{~s}, \mathrm{Ru} 3 \mathrm{~d}_{5 / 2}$ and $\mathrm{Ru} 3 \mathrm{p}_{3 / 2}$ regions of 10

\section{Catalytic Activity: Alcohol oxidation-Wittig olefination}

Embedded ruthenium nanoparticles in aluminum oxyhydroxide have been reported as catalysts for the one-pot alcohol oxidationWittig reaction producing $\alpha, \beta$-unsaturated esters. ${ }^{13}$ in this report 15 the overall reaction is mediated by the aldehyde. This reaction was selected as a test to evaluate the activity of the new catalysts. Interestingly, we were unable to oxidize benzyl alcohol to benzaldehyde using SRuNP in toluene at $80^{\circ} \mathrm{C}$ under oxygen atmosphere; however in presence of 20 methyl(triphenylphosphoranylidene) acetate the reaction proceeded with formation of the $\alpha, \beta$-unsaturated ester methyl cinnamate (Scheme 1). This suggests that in contrast with the literature example, our catalysts can yield Wittig products without free aldehyde mediation.

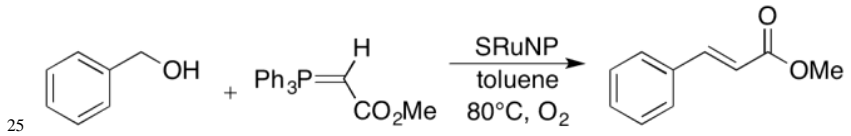

Scheme 1. Alcohol oxidation-Wittig olefination

The reaction does not occur without using SRuNP (Table 2, entry 1) or when only the support is used as catalyst (Table 2, entry 2). The effects of organic solvent, temperature, time of
30 reaction, atmosphere, and $\mathrm{Ru} \%$ on the yield and stereoselectivity were investigated using Ru@MCM, as shown on Table 2.

Table 2. Reaction of benzyl alcohol with methyl (triphenylphosphoranylidene) acetate ${ }^{\mathrm{a}}$

\begin{tabular}{|c|c|c|c|c|c|c|}
\hline \# & $\begin{array}{c}\text { Catalyst } \\
(\mathrm{mol} \\
\%)\end{array}$ & Solvent & $\begin{array}{c}\text { Conditions } \\
\text { Time }\end{array}$ & $\begin{array}{r}\mathrm{Con}_{\mathrm{v}} \\
(\%)^{\mathrm{b}}\end{array}$ & $\begin{array}{r}\text { Product } \\
\text { Yield } \\
(\%)^{\mathrm{c}}\end{array}$ & $\mathrm{E} / \mathrm{Z}$ ratio $^{\mathrm{d}}$ \\
\hline 1 & none & Toluene & $\begin{array}{c}80^{\circ} \mathrm{C}, \\
\mathrm{O}_{2} / 18 \mathrm{~h}\end{array}$ & 0 & - & - \\
\hline 2 & $\begin{array}{l}\text { MCM- } \\
\text { APTES }\end{array}$ & Toluene & $\begin{array}{c}80^{\circ} \mathrm{C} \\
\mathrm{O}_{2} / 18 \mathrm{~h}\end{array}$ & 0 & - & - \\
\hline 3 & 0.4 & $\mathrm{CCl}_{4}$ & $\begin{array}{c}80^{\circ} \mathrm{C} \\
\mathrm{O}_{2} / 18 \mathrm{~h}\end{array}$ & 32 & 27 & $\begin{array}{c}\text { not } \\
\text { quantified }\end{array}$ \\
\hline 4 & 0.4 & Toluene & $\begin{array}{c}80^{\circ} \mathrm{C}, \\
\mathrm{O}_{2} / 18 \mathrm{~h}\end{array}$ & 49 & 49 & $11: 1$ \\
\hline 5 & 0.4 & DMF & $\begin{array}{c}80^{\circ} \mathrm{C}, \\
\mathrm{O}_{2} / 18 \mathrm{~h}\end{array}$ & 12 & 12 & $11: 1$ \\
\hline 6 & 0.4 & THF & reflux/18h & 0 & - & - \\
\hline 7 & 0.4 & Toluene & $\begin{array}{l}110^{\circ} \mathrm{C} \\
\mathrm{O}_{2} / 18 \mathrm{~h}\end{array}$ & 34 & 31 & $10: 1$ \\
\hline \multirow[t]{5}{*}{8} & 0.4 & Toluene & $110^{\circ} \mathrm{C}$ & 10 & not & not \\
\hline & & & $\mathrm{N}_{2} / 18 \mathrm{~h}$ & & \multicolumn{2}{|c|}{ quantified quantified } \\
\hline & 9 & 0.4 Tolu & $\begin{array}{r}\text { ene } 80^{\circ} \mathrm{C} \\
\text { air } / 18 \mathrm{~h}\end{array}$ & 48 & $40^{\mathrm{d}}$ & $11: 1$ \\
\hline & 10 & 1.2 Tolue & $\begin{array}{r}80^{\circ} \mathrm{C} \\
\mathrm{O}_{2} / 24 \mathrm{~h}\end{array}$ & 70 & 61 & $17: 1$ \\
\hline & 11 & 1.2 Tolue & $\begin{array}{r}80^{\circ} \mathrm{C} \\
\mathrm{O}_{2} / 48 \mathrm{~h}\end{array}$ & 100 & 81 & $16: 1$ \\
\hline
\end{tabular}

${ }^{a}$ Benzyl alcohol (0.1 mL, $\left.1.0 \mathrm{mmol}\right)$, methyl(triphenylphosphoranylidene) 35 acetate $(334 \mathrm{mg}, 1.0 \mathrm{mmol})$ and the state amount of Ru@MCM in $2 \mathrm{~mL}$ of solvent. ${ }^{b}$ Quantification of unreacted benzyl alcohol. ${ }^{\mathrm{c}}$ The product methyl cinnamate was determined by GC using $1,1^{\prime}$ 'binaphthyl as internal standard, error $<5 \%$. ${ }^{\mathrm{d}}$ Together with benzyl cinnamate product of transesterification.

${ }_{40}$ Higher conversions were obtained when non-polar solvents such as toluene and $\mathrm{CCl}_{4}$ were used. Very low yield was obtained when DMF was used (Table 2 entry 5), while in THF no conversion was achieved even under reflux for $18 \mathrm{~h}$ (Table 2 entry 6). Increasing the temperature from 80 to $110^{\circ} \mathrm{C}$ was not 45 reflected in an increase of the conversion (Table 2, entries 4 and

7). When the reaction was performed under air, the byproduct benzyl cinnamate was obtained together with a similar yield of the desired methyl cinnamate (Table 2, entry 9); whereas, under $\mathrm{N}_{2}$ very little reaction was observed even after increasing the 50 temperature (Table 2, entry 8 ). When the percentage of Ru was increased up to $1.2 \%$ the conversion enhanced up to $70 \%$ after $24 \mathrm{~h}$; nevertheless quantitative conversion could be obtained after $48 \mathrm{~h}$ (Table 2, entries 10, 11).

From the data in Table 2, the reaction leads always to the E55 methyl cinnamate as the preferred isomer. Further, in order to evaluate the scope of this reaction, different primary alcohols were used as shown in Table 3. 
Table 3. Reaction of different primary alcohols with methyl(triphenylphosphoranylidene) acetate $^{\mathrm{a}}$

$\begin{gathered}\text { Product } \\ \text { yield \% }\end{gathered}$
$\begin{gathered}\mathrm{E} / \mathrm{Z} \\ \text { ratio }^{\mathrm{b}}\end{gathered}$
(1)

${ }^{\text {a }}$ Primary alcohol $(0.1 \mathrm{~mL}, 1.0 \mathrm{mmol})$,

5 methyl(triphenylphosphoranylidene) acetate $(334 \mathrm{mg}, 1.0 \mathrm{mmol})$ and 1.2 mol \% of Ru@MCM in 2 mL of toluene, under oxygen atmosphere for 24 h. ${ }^{\mathrm{b}}$ The product yields were determined by gas chromatography using 1,1 '-binaphthyl as internal standard, error $<5 \%$.

Finally, the nature of the silica support as well as the 10 recyclability in the reaction of the benzyl alcohol with methyl (triphenylphosphoranylidene)acetate was tested (Table 4). MCM41 and SBA-15 type silica showed the same conversion. Nevertheless, Ru@MCM material was more selective toward the E-methylcinnamate isomer. This is may reflect the smaller pore 15 size in the MCM type silica, i.e., $3 \mathrm{~nm}$, against the $9 \mathrm{~nm}$ pore size of the SBA-15 type silica. Moreover, HMS solids showed lower conversions due to their porosity disorder. With regard to the recyclability, all the catalysts conserved $50 \%-60 \%$ of its original activity upon second use (Table 4 ).

${ }_{20}$ Table 4. Recyclability of SRuNP in the reaction of the benzyl alcohol with methyl (triphenylphosphoranylidene) acetate.

\begin{tabular}{cccc}
\hline Catalyst & Conversion $(\%)$ & E/Z ratio & $\%$ reuse \\
\hline Ru@MCM & 70.0 & $17: 1$ & 50 \\
Ru@SBA & 70.0 & $11: 1$ & 60 \\
Ru@HMS & 55.0 & $17: 1$ & 60 \\
\hline
\end{tabular}

Although the mechanism of the alcohol oxidation-Wittig olefination has not been investigated in detail in the absence of 25 the methyl(triphenylphosphoranylidene) acetate the benzaldehyde was not detected when the reaction of the catalyst and alcohol was attempted.

\section{Discussion}

30 Metal nanoparticles are usually prepared by reduction of the aqueous salt in the presence of a protective agent to prevent aggregation. Literature reports indicate that RuNP could be obtained using polyols as reductants and PVP as stabilizer under microwave heating ${ }^{21}$ or simply refluxing. ${ }^{22}$ On the other hand,

35 amines have been reported as useful reducing agents in the formation of AuNPs from $\mathrm{HAuCl}_{4}$, acting also as protecting agents. ${ }^{23}$ Here we have demonstrated that the two concepts can be applied to the synthesis of new hybrid heterogeneous materials based on RuNP. In fact, APTES anchored to the surface of 40 MCM-41, SBA-15 or HMS silicas acted as both a reducing and protecting agent. The protocol developed is simple and mild since stirring for just 1 hour at room temperature was enough to reduce the $\mathrm{Ru}(\mathrm{III})$ salt and incorporate the resulting RuNP into the mesoporous silica solids. The properties of the materials 45 obtained, Ru@MCM, Ru@SBA and Ru@HMS include defined particle size and narrow distribution. ${ }^{24}$ TEM images (Figure 2) showed that RuNP are embedded into the pores of the solids, thus inhibiting the agglomeration of the nanoparticles and the subsequent loss of activity. Incorporating of the RuNP into the 50 silica mesopores led to the expected decrease of the surface of the final solid. Nevertheless, $740 \mathrm{~m}^{2} / \mathrm{g}$ for Ru@MCM was much higher than than the $424.7 \mathrm{~m}^{2} / \mathrm{g}$ reported for the analogous heterogeneous $\mathrm{Ru}(0)$ catalyst $\mathrm{Ru} / \mathrm{AlO}(\mathrm{OH}) .{ }^{25}$ XPS measurements performed on the all new materials, including the MCM-APTES 55 were consistent with the presence of $\mathrm{Ru}(0)$. As shown in Figure 4, the bands at $280.4 \mathrm{eV}$ and $462.0 \mathrm{eV}$ can be attributed to the $\mathrm{Ru}$ $3 \mathrm{~d}_{5 / 2}$ and $\mathrm{Ru} 3 \mathrm{p}_{3 / 2}$, respectively. ${ }^{16,22}$

Although the Wittig reaction is used worldwide as the most common method to prepare alkenes the mechanism involved is 60 still under scrutiny. ${ }^{12}$ In particular, ruthenium complexes have already been reported as catalysts for the Wittig reaction starting from alcohols through a temporarily oxidized alcohol. ${ }^{26,27}$ On the other hand, metal nanoparticles are not very common among the catalysts used for the one-pot olefination starting from alcohols;

${ }_{65}$ in fact only NiNPs have been reported so far and the reaction was claimed to proceed without a standard redox step. ${ }^{28,29}$ In this paper, beyond preparing efficient Wittig catalysts by very mild routes, it is worth noting that our evidence shows that the free aldehyde does not mediate the reaction, as these catalysts are 70 unable to oxidize alcohols to the corresponding aldehydes. Perhaps association of the alcohol at the ruthenium site occurs,

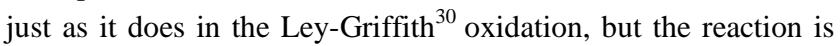
aborted if the Wittig reagent is not present. The nature of the support as well as the highly dispersed anchored RuNP may play 75 a crucial role since embedded RuNP in aluminum oxyhydroxide have been reported as catalysts for the same reaction producing $\alpha, \beta$-unsaturated esters mediated by the aldehyde. ${ }^{13}$ The chemistry described here bears some resemblance to 'borrowing hydrogen' type mechanisms, ${ }^{26,29,31,32}$ however, in this case the aldehyde 80 (the product of borrowing hydrogen from an alcohol) is never isolated or "free". Further, the borrowed hydrogen is not returned to the nascent double bond, but presumably to oxygen (that is essential) to form water.

From the data shown in Table 2, the reaction leads always to the ${ }_{85}$ E-methyl cinnamate as the preferred isomer. Higher conversions were obtained when non-polar solvents such as toluene and $\mathrm{CCl}_{4}$ 
were used. A very low yield was obtained when DMF (Table 2 entry 5) was used while in THF no conversion was achieved even under reflux for $18 \mathrm{~h}$ (Table 2 entry 6). Increasing the temperature from 80 to $110^{\circ} \mathrm{C}$ was not reflected in an increase of 5 the conversion (Table 2, entries 4 and 7). When the reaction was performed under air, the byproduct benzyl cinnamate was obtained together with a similar yield of the desired methyl cinnamate (Table 2, entry 9); whereas, under $\mathrm{N}_{2}$ almost no reaction took place, even after increasing the temperature (Table ${ }_{10} 2$, entry 8 ). When the percentage of $\mathrm{Ru}$ was increased up to $1.2 \%$ the conversion increased up to $70 \%$ after $24 \mathrm{~h}$; nevertheless quantitative conversion could be obtained after $48 \mathrm{~h}$ (Table 2, entries 10,11). Blank experiments showed that the reaction does not occur without using SRuNP (Table 2, entry 1) or when only 15 the support was tested as a possible catalyst (Table 2, entry 2).

Table 3 shows that the steric hindrance plays an important role in the yield and selectivity of the reaction. The OMe substituent was evaluated in the ortho, meta and para positions of benzyl alcohol. When the OMe substituent was in para position the 20 donating effect of the substituent achieved quantitative conversion and excellent selectivity as shown by the $\mathrm{E} / \mathrm{Z}$ ratio. However, the yield and selectivity decreased as steric hindrance increased (Table 3 entries 2 and 3). Even more, the very sterically hindered anthracene-9-methanol gave the desired product in only ${ }_{25} 40 \%$ yield being the E/Z ratio as low as 2:1 (Table 3, entry 5). The effect of an electron-withdrawing group in the para position decreases the yield and the $\mathrm{E} / \mathrm{Z}$ ratio (Table 3 , entry 4 ). In addition, in Table 3, entry 6 , the ester was obtained, but eventually the alkene was hydrogenated to afford the saturated 30 product, in accordance with previous results using $[\mathrm{Ir}(\mathrm{COD}) \mathrm{Cl}]_{2}{ }^{31}$ Table 4 showed that MCM-41 and SBA-15 type silica showed the same conversion. Nevertheless, Ru@MCM material was more selective to the E-methylcinnamate isomer. This is may reflect the smaller pore size in the MCM type silica, 35 i.e., $3 \mathrm{~nm}$, against the $9 \mathrm{~nm}$ for SBA-15 type silica. Moreover, HMS solids showed lower conversions due to their porosity disorder. With regard to the recyclability, all the catalysts conserved $50 \%-60 \%$ of its original activity upon second use.

\section{Conclusion}

${ }_{40}$ Three new materials based on MCM-41, SBA-15 and HMS type silica containing RuNP covalently attached to the structure have been prepared under very mild conditions; stirring a $\mathrm{Ru}(\mathrm{III})$ salt at room temperature in the presence of the solid grafted with APTES led to the formation of RuNP of $3 \mathrm{~nm}$ average size highly 45 dispersed into the channels of the mesoporous silicas. The materials exhibited high catalytic activity in the alcohol-oxidation Wittig olefination of different benzyl alcohols. Interestingly, the reaction does not appear to involve the intermediacy of the aldehyde, the usual Wittig reagent.

\section{${ }_{50}$ Acknowledgement}

Thanks are due to the Natural Sciences and Engineering Council of Canada and the Canadian Foundation for Innovation for generous support. M.L. Marin thanks the financial support of the Universitat Politécnica de Valencia (Programa de Apoyo a la ${ }_{55}$ Investigación y Desarrollo). Thanks are due to Dr. Yun Liu for advice on XPS interpretation.

\section{Notes and references}

${ }^{a}$ Department of Chemistry and Centre for Catalysis Research and Innovation, University of Ottawa, 10 Marie Curie, Ottawa, K1N 6N5, ${ }_{60}$ Canada E-mail: scaiano@photo.chem.uottawa.ca

${ }^{b}$ Instituto Universitario Mixto de Tecnología Química (UPV-CSIC), Universitat Politècnica de València. Avenida de los Naranjos s/n, 46022 Valencia. Spain

65 1. G. C. F. Cavani, S. Perathoner, F. Trifiro, Sustainable Industrial Chemistry, Wiley-VHC, Weinheim, 2009.

2. V. Hulea, D. Brunel, A. Galarneau, K. Philippot, B. Chaudret, P. J. Kooyman and F. Fajula, Microporous Mesoporous Mater., 2005, 79, 185.

70 3. A. Taguchi and F. Schüth, Microporous Mesoporous Mater., 2005, $77,1$.

4. D. Trong On, D. Desplantier-Giscard, C. Danumah and S. Kaliaguine, Appl. Cat. A: Gen., 2001, 222, 299; Y. Wu, L. Zhang, G. Li, C. Liang, X. Huang, Y. Zhang, G. Song, J. Jia and C. Zhixiang,

75 Mater. Res. Bull., 2001, 36, 253.

5. J. Garcia-Martinez, N. Linares, S. Sinibaldi, E. Coronado and A. Ribera, Microporous Mesoporous Mater., 2009, 117, 170.

6. A. I. Carrillo, J. García-Martínez, R. Llusar, E. Serrano, I. Sorribes, C. Vicent and J. Alejandro Vidal-Moya, Microporous Mesoporous 80 Mater., 2012, 151, 380.

7. E. Iglesia, S. L. Soled, R. A. Fiato and G. H. Via, J. Catal., 1993, 143,345 .

8. D. P. VanderWiel, M. Pruski and T. S. King, J. Catal., 1999, 188, 186.

85 9. V. Mazzieri, F. Coloma-Pascual, A. Arcoya, P. C. L'Argentière and N. S. Figoli, Appl. Surf. Sci., 2003, 210, 222.

10. J. Zhang, H. Xu, Q. Ge and W. Li, Catal. Comm., 2006, 7, 148.

11. F. Su, L. Lv, F. Y. Lee, T. Liu, A. I. Cooper and X. S. Zhao, J. Am. Chem. Soc., 2007, 129, 14213.

90 12. P. A. Byrne and D. G. Gilheany, Chem. Soc. Rev., 2013, 42, 6670.

13. E. Y. Lee, Y. Kim, J. S. Lee and J. Park, Eur. J. Org. Chem., 2009, 2943.

14. Z. Luan, M. Hartmann, D. Zhao, W. Zhou and L. Kevan, Chem. Mater., 1999, 11, 1621.

95 15. W. Zhang, T. R. Pauly and T. J. Pinnavaia, Chem. Mater., 1997, 9 , 2491.

16. I. Kusunoki and Y. Igari, Appl. Surf. Sci., 1992, 59, 95; H. Zarrin, D. Higgins, Y. Jun, Z. Chen and M. Fowler, J. Phys. Chem. C, 2011, $115,20774$.

100 17. A. I. Carrillo, E. Serrano, R. Luque and J. G. Matinez, Chem. Comm., 2010, 46, 5163.

18. K. R. Chary and C. Srikanth, Catal. Lett., 2009, 128, 164.

19. J. Chen, J. Zhou, R. Wang and J. Zhang, Ind. Eng. Chem. Res., 2009, $48,3802$.

105 20. A. I. Carrillo, N. Linares, E. Serrano and J. García-Martínez, Mat. Chem. Phys.s, 2011, 129, 261.

21. W. Tu and H. Liu, J. Mat. Chem., 2000, 10, 2207.

22. X. Yan, H. Liu and K. Y. Liew, J. Mat. Chem., 2001, 11, 3387.

23. J. D. S. Newman and G. J. Blanchard, Langmuir, 2006, 22, 5882.

11024 . D. T. Marquez, A. I. Carrillo and J. C. Scaiano, Langmuir, 2013, 29, 10521.

25. W.-H. Kim, I. S. Park and J. Park, Org. Lett., 2006, 8, 2543.

26. M. G. Edwards, R. F. R. Jazzar, B. M. Paine, D. J. Shermer, M. K. Whittlesey, J. M. J. Williams and D. D. Edney, Chem. Comm., 2004, 11590.

27. S. Burling, B. M. Paine, D. Nama, V. S. Brown, M. F. Mahon, T. J. Prior, P. S. Pregosin, M. K. Whittlesey and J. M. J. Williams, Journal of the American Chemical Society, 2007, 129, 1987.

28. F. Alonso, P. Riente and M. Yus, Eur. J. Org. Chem., 2009, 2009, $120 \quad 6034$.

29. F. Alonso, P. Riente and M. Yus, Acc. Chem. Res., 2011, 44, 379.

30. W. P. Griffith, S. V. Ley, G. P. Whitcombe and A. D. White, J. Chem. Soc., Chem. Comm., 1987, 1625. 
31. P. J. Black, M. G. Edwards and J. M. J. Williams, Eur. J. Org. Chem., 2006, 2006, 4367.

32. T. D. Nixon, M. K. Whittlesey and J. M. J. Williams, Dalton Trans., $2009,753$. 